\title{
Laser Scanner Versus Stereophotogrammetry: A Three-Dimensional Quantitative Approach for Morphological Analysis of Pubic Symphysis
}

\author{
Valentina PUCCIARELLI ${ }^{1}$, Daniele Maria GIBELLI ${ }^{1,2}$, Marina CODARI ${ }^{1}$, Francesca Maria Emilia \\ RUSCONI ${ }^{1}$, Annalisa CAPPELLA ${ }^{1,2,}$ Cristina CATTANEO ${ }^{1,2}$, Chiarella SFORZA ${ }^{1 *}$ \\ ${ }^{1}$ LAFAS, Laboratorio di Anatomia Funzionale dell'Apparato Stomatognatico, Dipartimento \\ di Scienze Biomediche per la Salute, Università degli Studi di Milano, Milano, Italy; \\ ${ }^{2}$ LABANOF, Laboratorio di Antropologia e Odontologia Forense, Dipartimento \\ di Science Biomediche per la Salute, Università degli Studi di Milano, Milano, Italy
}

DOI: $10.15221 / 16.080 \quad$ http://dx.doi.org/10.15221/16.080

\begin{abstract}
The reconstruction of biological profile is a crucial step for personal identification of unknown decedents, both in forensic and archaeological context. At this purpose, determination of age at death is a very important issue. Among the skeletal regions used for estimating it, the analysis of pubic symphysis by Suchey-Brooks method remains the most reliable aging technique. In the last years, technologies involving 3D image acquisition have acquired a growing importance in anthropology and may provide a relevant help in diffusion and sharing of skeletal specimens. In particular, among all the new available instruments, laser scanners and stereophotogrammetric systems are particularly useful to obtain 3D reconstructions of the pubic symphyseal surface. Furthermore, the possibility to create digital models of these bony structures allows researchers to easily and fast share their datasets worldwide, avoiding any possible damage to the real sample. This study aims at verifying the technical reproducibility of $3 \mathrm{D}$ acquisitions, performed by laser scanner and stereophotogrammetry, as a preliminary study to possible assessment of differences in age at death estimation. Since accuracy and reproducibility of these two instruments have already been evaluated, the study aims at comparing them in order to verify if there are some differences on relevant measurements obtained from the analysis of the 24 commercial casts (by Diane France). These 24 symphyseal casts ( 12 female and 12 male) were scanned by a dental laser scanner (Dental Wings series 3, Dental Wings Inc., Montreal, Canada) and a stereophotogrammetric system (Vectra 3D, Canfield Scientific, Fairfield, NJ). For each sample two scans were performed by the same tool and some measurements (symphyseal surface length, symphyseal surface area and billowing area) were taken twice on digitized surfaces, by the same operator. The second assessment was performed 15 days after the first one.

Repeatability of operator measurements on different scans was assessed by paired Student t-test, or Mann-Whitney test, depending on data distribution. Linear regression was performed as well. To compare the two instruments, Bland-Altman analysis was carried out for billowing measurements. Results show no significant difference $(p>0.05)$ in identification of the same parameter on different scans through both instruments and no significant difference between billowing areas, measured through laser scanner or stereophotogrammetry.

In conclusion, data suggest that both 3D image acquisition systems may provide a technically reliable reproduction of pubic symphysis. However, the technical reproduction of skeletal specimens does not mean that the 3D scans are suitable for age estimation, which remains, in anthropological practice, a morphological procedure and requires a specific experience of the operator. Further studies are required to validate the opportunity to use this approach for anthropological age at death estimation.
\end{abstract}

Keywords: pubic symphysis, laser scanner, stereophotogrammetry

\section{Introduction}

Determination of age at death is a very important issue in the reconstruction of the biological profile of unknown deceased persons, both in forensic anthropology and archeology [1,2]. Several skeletal regions are used for estimating age at death in adults, like pubic symphysis, sternal rib ends, auricular surface from os coxae, cranial sutures, but the analysis of pubic symphysis through Suchey-Brooks method (1990) remains the most highly favored aging technique [3-6].

In particular, Garvin et al., found, through an electronic questionnaire distributed to all members of the Physical Anthropology Section of the American Association of Forensic Sciences (AAFS), that $78 \%$ of

* chiarella.sforza@unimi.it; +39 02503 15387; www.scibis.unimi.it 
them considered the pubic symphysis as the preferred region for age at death estimation [7]. Indeed, one of the advantages of pubic symphysis, in contrast with other skeletal regions, is that the rate of its age-related degeneration does not seem to be influenced by physical or occupational activity, rendering it helpful to estimate age at death of individuals with an unknown identity. This characteristic was recently reinforced by a paper by Campanacho et al., that did not support the idea of a more pronounced pubic symphysis degeneration in individuals affected by occupational or physical activity [8]. For these reasons, among all the available procedures, the analysis of pubic symphyses through Suchey-Brooks method proved to be accurate and the most widely used [9-11].

The method involves a visual and qualitative procedure founded on the comparison between the test and a series of 24 commercial casts, designed to represent the couples of most representative morphology for the six male and female Suchey-Brooks age phases. These phases correspond to six different stages of morphological variation for each gender, from juvenile to adult/old age. The overall procedure relies on operator experience [3, 12-13].

If, on one hand, age at death estimation is a qualitative/manual procedure, on the other hand, in the last decades, the use of digital technologies in anthropology gained a lot of importance. For example, $\mathrm{U}$-Young et al., used CT images of upper and lower limbs to evaluate the sexual dimorphism through discriminant analysis [14]. CT and laser scanner acquisitions were used for matching pairs of bony models, using a mesh-to-mesh comparison, in order to help recognition processes during mass disasters. Three-dimensional approaches on symphyseal surface scans were also performed [15]. Examples are the studies by Villa et al. who made a comparison among pubic bone scans obtained using CTs and laser scanners and recently studied the symphyseal curvature variation on the same kind of models $[16,17]$. However, to the best of our knowledge, no one ever evaluated symphyseal casts with stereophotogrammetry.

Recently, a comparison of area measurements from nasal plaster casts obtained by a laser scanner and a stereophotogrammetric system showed no significant differences among the values measured with both instruments [18]. Therefore, the aim of the current study is to compare these two instruments and verify their capability to reproduce relevant measurements, obtained from the analysis of symphyseal reference casts. This will pave the way to the possibility of investigation about the future applications of these technologies on age at death estimation from pubic symphysis..

\section{Method}

\subsection{Sample and instruments}

The sample was composed by the 24 casts by Diane France $\odot$, which are considered the current standards for age estimation of unknown death specimens based on Suchey-Brooks method. They include 12 male and 12 female pubic bone models.

These models are based on a real sample, obtained by Suchey and Brooks from 739 males and 273 females, aged 14 to 99 years. Real age at death was ascertained from birth or death certificate and the bones were gently prepared, so high quality specimens were obtained [3].

Diane France $\odot$ models were acquired using two optical surface scanners that can provide a digital three-dimensional reconstruction of objects: a stereophotogrammetric system (VECTRA M3, Canfield Scientific Inc, Fairfield, NJ, USA) and a laser scanner (Dental wings series 3, Dental wings, Montreal, Canada). The stereophotogrammetric system has a short capture time $(3.5 \mathrm{~ms})$ and a geometry resolution of $1.2 \mathrm{~mm}$; moreover, it can reproduce colored reconstructions. The laser scanner is an instrument originally designed for dental applications, with an accuracy of $15 \mu \mathrm{m}$. The digitized images obtained through this instrument do not have a colored texture. An example of the digitized casts, obtained with both instruments, is depicted in Figure 1.

\subsubsection{Stereophotogrammetric acquisition procedure}

Before the stereophotogrammetric acquisition of the casts, a previous calibration of the instrument was performed. Calibration process is done using a calibration panel provided by the producer and takes about 2 minutes. Then, each model was fixed, using a little quantity of modeling clay, to a robust black cardboard. The positioning of the model was performed so that the ilio-pubic branch was firmly fastened to the cardboard. The cardboard was chosen with appropriate characteristics, in order to avoid any reflection from its surface that could interfere with the three-dimensional reconstruction. 


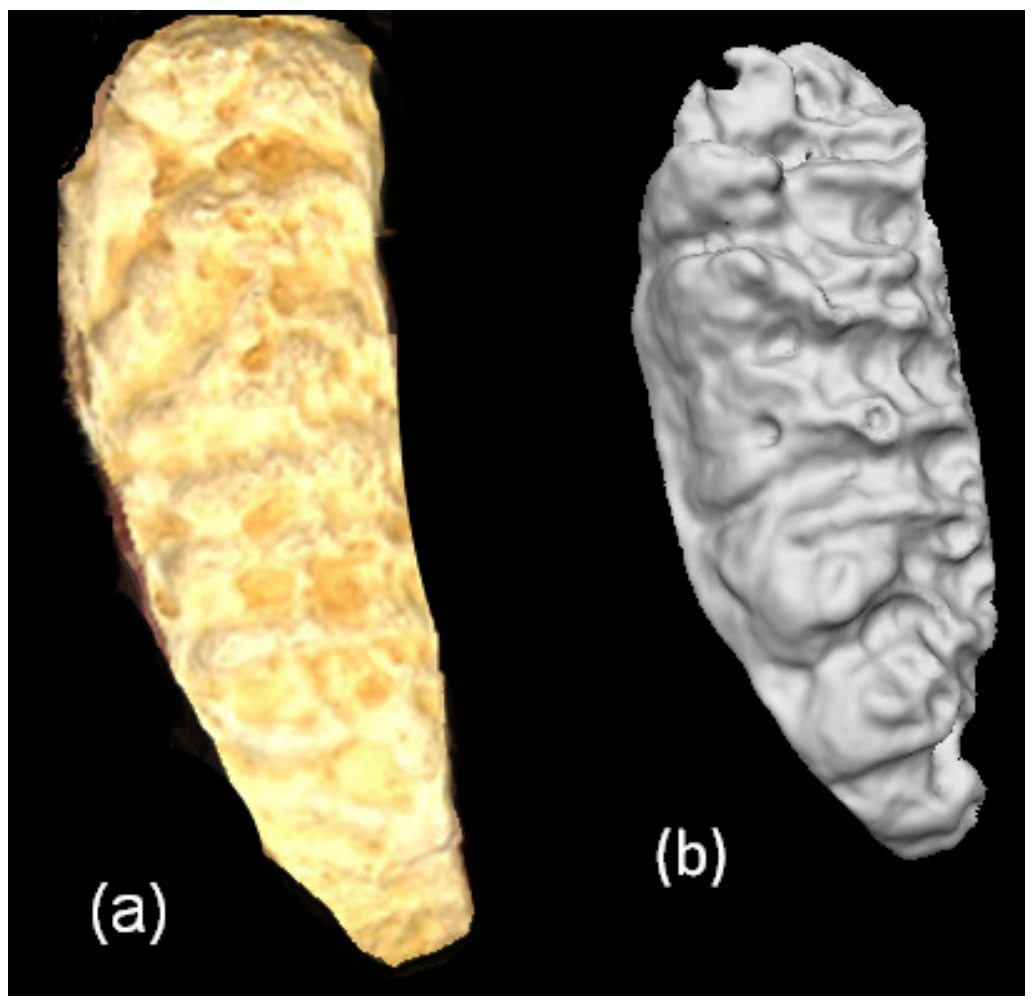

Fig.1. (a) Symphyseal surface scan obtained through a stereophotogrammetric system (male, phase $1 \mathrm{~A}$ of Suchey-Brooks age phases corresponding to an average age of 19.5 years, SD 2.6 years); (b) symphyseal surface scan obtained through a laser scanner (female, phase $1 \mathrm{~A}$, corresponding to an average age of 18.5 years, $S D 2.1$ years).

Each stereophotogrammetric acquisition was performed by two operators. The first, keeping the cardboard with the cast in the correct orientation (symphyseal surface towards the cameras) in order to obtain the $3 \mathrm{D}$ reconstruction of its surface; the other capturing the image. Correct acquisition was assessed for each model.

This procedure was repeated twice for all casts. All the 12 female and 12 male casts were digitized with this technique, for a total of 48 stereophotogrammetric reconstructions.

\subsubsection{Laser scanner acquisition procedure}

Before the scan, the instrument was calibrated through a calibration plate following producer's instructions. This process requires about 10 minutes.

Subsequently, each cast was positioned on the model holder. As for stereophotogrammetric acquisitions, the positioning of the models was performed so that the ilio-pubic branch was firmly fastened to the support, using some modeling clay. Scan was started and a region of interest (ROI) was selected. This region included symphyseal surface. Every scan was, then, checked for accuracy. Each model was acquired twice, for a total of 48 symphyseal three-dimensional reconstructions (24 for female casts and 24 for male casts).

\subsection{Image analysis}

All the digitized reconstructions of the symphyseal Diane France@ casts (obtained with both laser scanner and stereophotogrammetry) were analyzed using the Mirror Vectra Software (Canfield Scientific, Fairfield, NJ).

In particular, after the removal of the extra areas surrounding the symphyseal surface, the following measurements were taken on each scan:

1. Maximum symphyseal length [mm], defined as the vertical distance between the most superior and the most inferior point of the symphyseal surface

2. Symphyseal surface area $\left[\mathrm{cm}^{2}\right]$, defined as the area included inside the edges that delimit the articular surface

3. Billowing area $\left[\mathrm{cm}^{2}\right]$, defined as the region of symphyseal surface characterized by a corrugation made of transversal osseous crests. This feature is typical of juvenile age phases and is absent in elderly symphyses $[3,9,19]$. 
A total of 96 scans (48 stereophotogrammetric reconstructions and 48 laser scanner acquisitions) were assessed. An example of measurements, performed on a laser scanner 3D reconstruction, is provided in Figure 2.

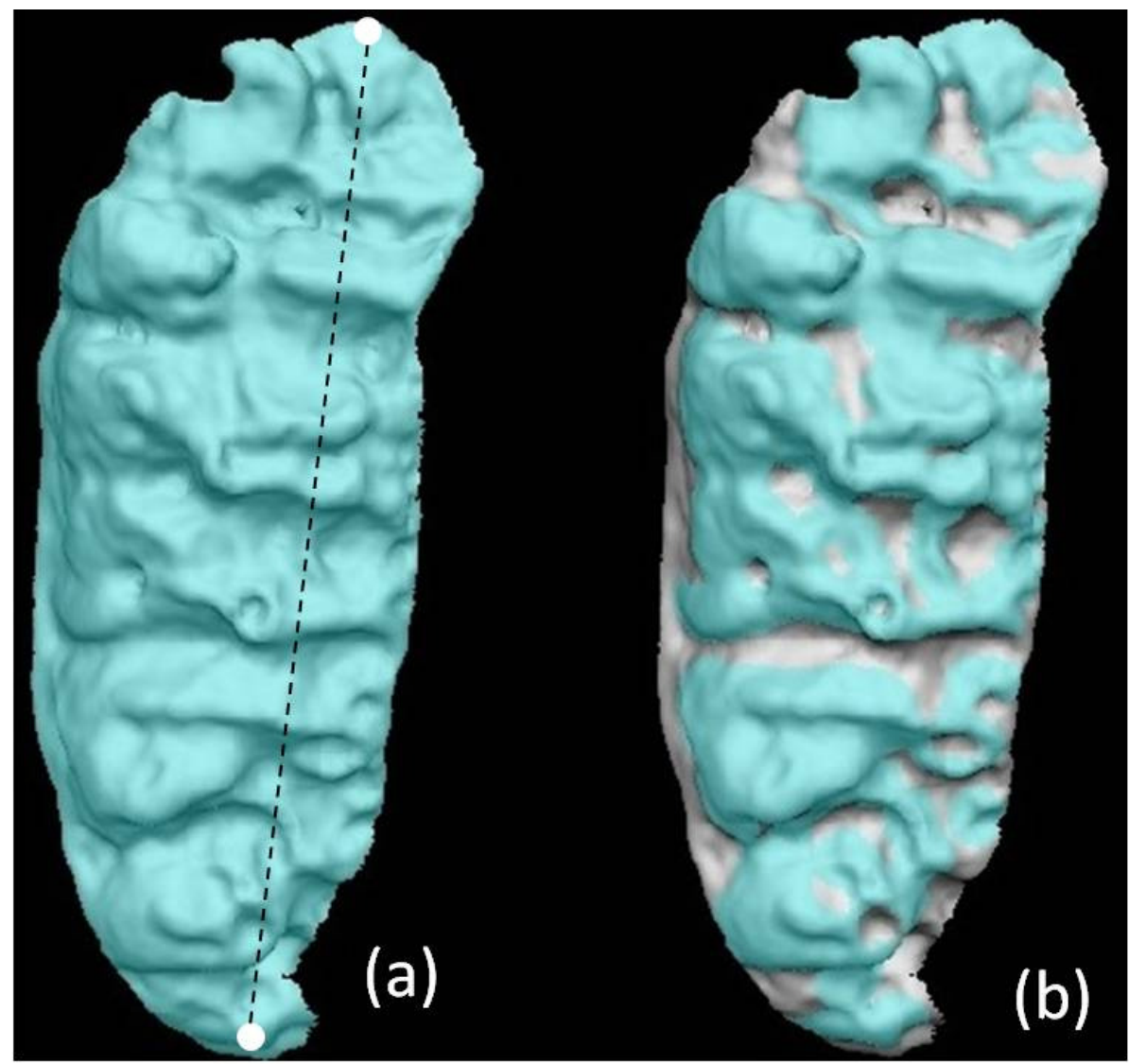

Fig.2. a) Symphyseal surface scan. Surface area is depicted In blue. The line indicates symphyseal maximum length. b) Symphyseal surface scan. Billowing area is indicated in blue. Both scans are obtained by a laser scanner.

\subsection{Statistical analysis}

In order to verify the repeatability of the performed measurements, symphyseal maximum length, symphyseal surface area and area of billowing were measured by the same operator twice, using the couples of scans obtained by the same instrument. The second assessment was performed 15 days after the first. Normality of the data was assessed using Jarque-Bera test. Depending on data distribution, repeatability of the operator was evaluated by paired Student t-test or Mann-Whitney test. Significance level was set at $0.05 \%$.

Linear regression analysis was performed on the repetitions of the measured parameters.

Billowing area was chosen as criterion to evaluate the differences on the acquisitions obtained with stereophotogrammetry and laser scanner. To compare the differences on billowing areas assessment, obtained through both techniques, Bland-Altman analysis was performed (separately for female and male casts). Repeatability coefficient and reproducibility were also calculated.

\section{Results}

\subsection{Measurements repeatability}

In this study, two stereophotogrammetric and two laser scanner acquisitions of each of the 24 Diane France@ casts were performed. The repeatability of the operator in assessing symphyseal length, symphyseal surface area and billowing area on different scans obtained with the same tool was assessed.

Results show no significant differences $(p>0.05)$ in the evaluation of these parameters on different 3D reconstructions, obtained through both the devices. Furthermore, $R^{2}$ values from linear regression analysis ranged between 0.96 and 0.99 for stereophotogrammetric acquisitions and between 0.95 and 0.99 for laser scans. 


\subsection{Comparison between stereophotogrammetry and laser scanner for billowing area measurements}

Figures 3 and 4 show Bland-Altman plots for comparison between the two instruments for the assessment of billowing areas on female and male casts, respectively. Details about bias, standard deviation (SD), repeatability coefficient (RC) and reproducibility are shown in Table 1.

Billowing values were not included for Suchey-Brooks age phase 6 because billowing is not detectable at this stage.

In female casts billowing area was measured with nearly identical values by both devices (bias was very close to 0 ). In contrast, male values were slightly overestimated by laser scanner relative to stereophotogrammetry. However, the coefficient of repeatability was low for both methods, indicating that differences lower than $7 \%$ and $15 \%$ for female and male casts respectively must not be considered real or due to the methods. Consequently, reproducibility was high for both female and male casts (93\% and $85 \%)$.

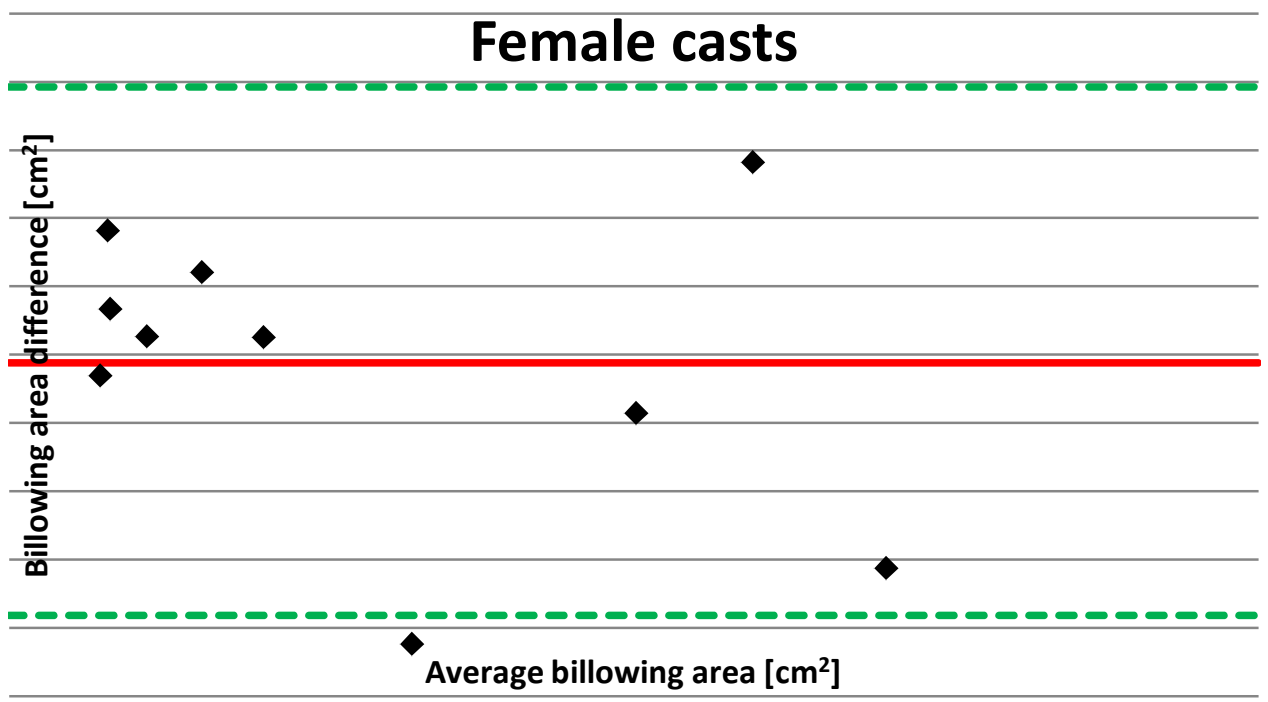

Fig.3. Bland and Altman plot for the billowing area measurements on female casts. Age phases 6 models were excluded. Continuous line indicates the average; dashed lines indicate the interval of agreement.

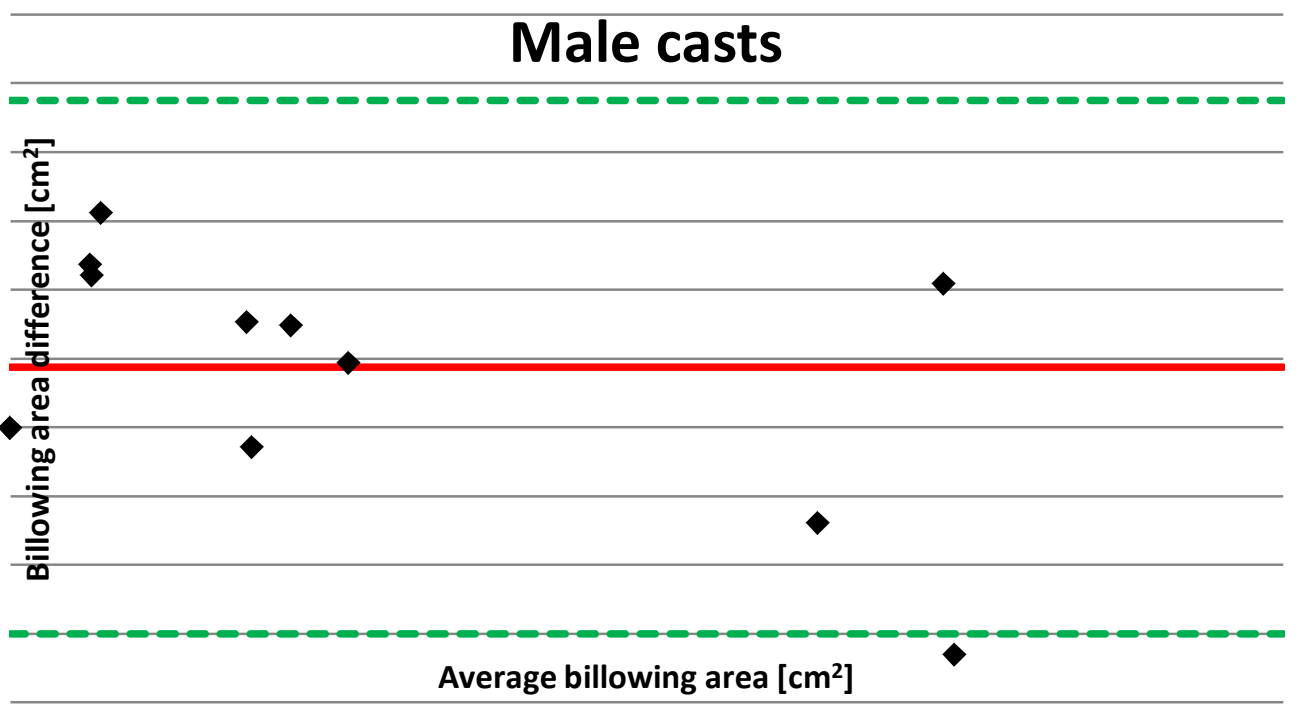

Fig.4. Bland and Altman plot for the billowing area measurements on male casts. Age phases 6 models were excluded. Continuous line indicates the average; dashed lines indicate the interval of agreement. 
Table 1. Results of Bland and Altman analysis. Bias, $S D, R C$ and reproducibility of billowing area measurements are reported for both female and male casts.

\begin{tabular}{lcc} 
& Female casts & Male casts \\
\hline Bias $\left[\mathrm{cm}^{2}\right]$ & -0.002 & -0.044 \\
SD $\left[\mathrm{cm}^{2}\right]$ & 0.038 & 0.098 \\
RC & $7 \%$ & $15 \%$ \\
Reproducibility & $93 \%$ & $85 \%$ \\
\hline
\end{tabular}

\section{Conclusions}

This study aimed at verifying the repeatability in performing some measurements, obtained from 3D reconstructions of symphyseal plaster casts, digitized through a stereophotogrammetric system and a laser scanner. Reproducibility of the techniques was also assessed, in order to test if these instruments were in good agreement for the performed measurements.

The reliability of both imaging systems has already been evaluated: a stereophotogrammetric system was assessed by Sforza et al., for accuracy and reproducibility on measurements obtained from stone casts of patients with unilateral cleft lip and palate. They found that the accuracy error was lower than $0.9 \%$ in the detection of the measurements of cubes and cylinders of different size [20]. Accuracy and reliability of measurements performed on geometric objects, facial and dental casts, digitized with a laser scanner, were also reported [21]. In our laboratory, we have recently found no differences in area assessment on nasal plaster casts using both instruments [18].

Even if during the last years, the new 3D image acquisition technologies have had a great impact on forensic science and anthropology, at the moment, age at death estimation from pubic symphysis remains, for its nature, a qualitative procedure, requiring the experience of a well trained operator [14-17]. Indeed, Sitchon et al., demonstrated that age at death estimation from pubic symphysis, using Suchey-Brooks method, may not exclude a physical/tactile examination [22]. Therefore, the visual assessment performed on two-dimensional digital images may be less adequate for a correct estimation. Anyway, they also showed that there is good concordance between the physical and visual assessment: this last can be especially helpful in successive re-assessments [22].

Starting from these considerations and from our successful comparison between the two optical scanners [18], our study wanted to verify the technical capability of stereophotogrammetry and laser scanner to reliably reproduce symphyseal surface morphology.

We particularly focused on the assessment of billowing area on symphyseal casts. Billowing, indeed, is an important parameter used by forensic anthropologists to evaluate age at death on juvenile samples. Furthermore, it has been showed that billowed surfaces can be very accurately represented by stereophotogrammetric systems and laser scanners for measuring agricultural soil micro-reliefs. [23]. This, indeed, could be a preliminary starting point to, successively, validate the possibility to use these instruments for anthropological age at death estimation. Indeed, three-dimensional reconstructions, such as those obtained from stereophotogrammetry and laser scanner, offer the possibility to rotate the acquired image on all axes, and this could help in overcoming the technical impairment of two-dimensional digital assessment reducing the limitation due to the lack of physical contact [22, 24]. Moreover, digital optical systems like stereophotogrammetry and laser scanners are less expensive and more diffuse than volumetric instruments such as CT and MR [25]. This allows their use even in laboratories which do not have enough resources to afford more expensive technologies. Furthermore, given their agreement on the performed measurements, they can also be exchanged each other, depending on the laboratory availability and possibilities [18].

Clearly the metrical assessment of billowing on 3D scans is not sufficient to provide the basis for novel methods of age estimation based on the pubic symphysis, for two reasons: first, billowing is just a feature among those usually included in a morphological assessment for age estimation, and mainly concerns young subjects. Other characteristics are included, such as the elevation of symphyseal edge, depression of articular surface, micro- and macro-porosity of the symphyseal surface, and some of them still lack methods for metrical assessment. Secondarily, the detection of billowing and therefore its measurement is subjective, as it is a manual procedure, and depends upon the accuracy of 3D model. For this reason a metrical method for assessing pubic symphysis is yet to come: however the present study verified that laser scanner and stereophotogrammetry do provide an accurate 
reconstruction of such specimens, and therefore may allow researchers to provide a morphological assessment based on traditional morphological Suchey-Brooks method.

Additionally, these systems are easy to use and do not require a long time to train an expert operator. They can also facilitate the exchange of samples worldwide and their storage in digital archives, which preserve samples from damages [26]. Moreover, the possibility of creating online databases, as suggested by Tocheri et al., will be a "considerable promise for improvements in the education and training of physical anthropologists, and also in the way and manner physical anthropologists are able to conduct osteological aging research" [16]. In conclusion, the technical capability of stereophotogrammetry and laser scanners to accurately reproduce 3D symphyseal surfaces will give the opportunity for future validation of age at death estimation through Suchey-Brooks method on three-dimensional reconstructions obtained using CAM/CAD techniques and three-dimensional printers. Furthermore, the possibility of three-dimensionally printimg a sample, previously acquired through one of these optical systems, will be an opportunity for medical students to examine anatomy in depth, and to evaluate single anatomical variants without the limits due to "average" anatomical models [27]. This will also reduce damages to the real samples, favor the exchange among the researchers and allow the creation of digital learning platforms for anthropologists, forensic scientists and medical students.

\section{References}

[1] C. Merritt, "A test of Hartnett's revisions to the pubic symphysis and fourth rib methods on a modern sample", in Journal of Forensic Sciences, Vol. 59, No.3, 2014, pp. 703-701, http://dx.doi.org/10.1111/1556-4029.12380.

[2] D. Franklin, "Forensic age estimation in human skeletal remains: current concepts and future directions", in Legal Medicine, Vol. 12, No. 1, 2010, pp. 1-7, http://dx.doi.org/10.1016/..legalmed.2009.09.001.

[3] S. Brooks and JM. Suchey, "Skeletal age determination based on the os pubis: a comparison of the acsádi-nemeskéri and Suchey-Brooks methods", in Journal of Human Evolution, Vol. 5, No. 3, 1990, pp. 227-238, http://dx.doi.org/10.1002/10.1007/BF02437238.

[4] RS. Meindl and CO. Lovejoy, "Ectocranial suture closure: a revised method for the determination of skeletal age at death based on the lateral-anterior sutures", in American Journal of Physical Anthropology, Vol. 68, No. 1, 1985, pp. 57-66, http://dx.doi.org/10.1002/ajpa.1330680106.

[5] CO. Lovejoy et al, "Chronological metamorphosis of the auricular surface of the ilium: a new method for the determination of adult skeletal age at death", in American Journal of Physical Anthropology, Vol. 68, No. 1, 1985, pp. 15-28, http://dx.doi.org/10.1002/ajpa.1330680103 .

[6] MY. Işcan et al., "Metamorphosis at the sternal rib end: a new method to estimate age at death in white males", in American Journal of Physical Anthropology, Vol. 65, No. 2, 1984, pp. 147-156, http://dx.doi.org/10.1002/ajpa.1330650206 .

[7] HM. Garvin, and N. Passalacqua, "Current practices by forensic anthropologists in adult skeletal age estimation", in Journal of Forensic Sciences, Vol. 57, No. 2, 2012, pp. 427-433, http://dx.doi.org/10.1111/j.1556-4029.2011.01979.x .

[8] V. Campanacho et al., "Assessing the influence of occupational and physical activity on the rate of degenerative change of the pubic symphysis in portuguese males from the 19th to 20th century" in American Journal of Physical Anthropology, Vol. 148, No. 3, 2012, pp. 371-378, http://dx.doi.org/10.1002/ajpa.22059.

[9] C. Cattaneo and M. Grandi "Antropologia e odontologia forense: guida allo studio dei resti umani”, testo atlante. Monduzzi, 2004, pp. 166-167.

[10]SK. Warmlander and SB. Sholts, "Sampling and statistical considerations for the Suchey-Brooks method for pubic bone age estimation: Implications for regional comparisons", in Science \& Justice, Vol. 51, No. 3, 2011, pp. 131-134, http://dx.doi.org/10.1016/i.scijus.2010.12.001.

[11] C. Rissech, et al., "A comparison of three established age estimation methods on an adult spanish sample", in International Journal of Legal Medicine, Vol. 126, No. 1, 2012, pp. 145-155, http://dx.doi.org/10.1007/s00414-011-0586-1.

[12] M. Djurić et al., "Evaluation of the Suchey-Brooks method for aging skeletons in the Balkans" in Journal of Forensic Sciences, Vol. 52, No. 1, 2007, pp. 21-23, http://dx.doi.org/10.1111/j.1556-4029.2006.00333.x. 
[13]C. Villa et al., "Quantitative analysis of the morphological changes of the pubic symphyseal face and the auricular surface and implications for age at death estimation" in Journal of Forensic Sciences, Vol. 60, No.3, 2015, pp. 556-565, http://dx.doi.org/10.1111/1556-4029.12689.

[14]L. U-Young et al., "Sex determination using discriminant analysis of upper and lower extremities bones: new approach using the volume and surface are of digital model", in Forensic Science International, Vol. 235, 2015, pp. e1-4, http://dx.doi.org/10.1016/i.forsciint.2015.05.017.

[15] MA. Karell, et al., "A novel method for pair-matching using three-dimensional digital models of bone: mesh-to-mesh value comparison", in International Journal of Legal Medicine, 2016, http://dx.doi.org/10.1007/s00414-016-1334-3.

[16] MW. Tocheri, et al., "Three dimensional quantitative analyses of human pubic symphyseal morphology: can current limitations of skeletal aging methods be resolved" in American Journal of Physical Anthropology Vol. 155, (Suppl. 34), 2002.

[17]C. Villa et al, "Technical Note: Reliability of Suchey-Brooks and Buckberry-Chamberlain methods on 3D visualizations from CT and laser scans", in American Journal of Physical. Anthropology, Vol. 151, No. 1, 2013, pp. 158-163, http://dx.doi.org/10.1002/ajpa.22254.

[18] M. Codari et al., "Laser scanner compared with stereophotogrammetry for measurements of area on nasal plaster casts", in British Journal of Oral and Maxillofacial Surgery, Vol. 53, No.8, 2015, pp. 769-770, http://dx.doi.org/10.1016/j.bjoms.2015.05.007.

[19]N. Lottering et al., "Morphometric modeling of ageing in the human pubic symphysis: Sexual dimorphism in an Australian population", in Forensic Science International, Vol. 236, No. 195, 2014, pp. e1-11, http://dx.doi.org/10.1016/.j.forsciint.2013.12.041.

[20]C. Sforza et al., "Evaluation of a 3D stereophotogrammetric technique to measure the stone casts of patients with unilateral cleft lip and palate", in Cleft Palate Craniofacial Journal, Vol. 49, No. 4, 2012, pp. 477-483, http://dx.doi.org/10.1597/10-207.

[21]B. Kusnoto and CA. Evans, "Reliability of a 3D surface laser scanner for orthodontic applications", in American Journal of Orthodontics and Dentofacial Orthopedics, Vol. 122, No. 4, 2002, pp. 342-348, http://dx.doi.org/10.1067/mod.2002.128219.

[22] M. Sitchon, and R.Hoppa, "Assessing Age-Related Morphology of the Pubic Symphysis from Digital Images Versus Direct Observation," in Journal of Forensic Sciences, Vol. 50, No. 4, 2005, pp. 791-795, http://dx.doi.org/10.1520/JFS2004182.

[23]MA. Aguilar et al, "Off-the-shelf laser scanning and close-range digital photogrammetry for measuring agricultural soils microrelief", in Biosystem engeenering, Vol. 103, No.4, 2009, pp. 504-517, http://dx.doi.org/10.1016/..biosystemseng.2009.02.010.

[24]G. Lekakis, et al., "Three-Dimensional surface imaging and the continuous evolution of preoperative and postoperative assessment in rhinoplasty", in Facial Plastic Surgery, Vol. 32, No. 1, 2016, pp. 88-94, http://dx.doi.org/10.1055/s-0035-1570122.

[25] C. Sforza et al, "Soft-and-hard tissue facial anthropometry in three dimensions: what's new", in Journal of Anthropological Sciences, Vol. 91, 2013, pp. 59-84, http://dx.doi.org/10.4436/JASS.91007.

[26] SC. Kuzminsky and MS. Gardiner, "Three-dimensional laser scanning: potential uses for museum conservation and scientific research", in Journal of Archaeological Sciences, Vol. 39, No. 8, 2010, pp. 2744-2751, http://dx.doi.org/10.1016/j.jas.2012.04.020.

[27]Y. AbouHashem et al., "The application of 3D printing in anatomy education", in Medical Education Online, Vol. 20, No 29847, 2015, .http://dx.doi.org/10.3402/meo.v20.29847. 